\title{
Rasch-progrediente Glomerulonephritis
}

\author{
Pechula Thut, M ; Hauser, T
}

\begin{abstract}
Die rapid progrediente Glomerulonephritis (RPGN) ist als klinisches Syndrom durch die zeitliche Veränderung der Nierenfunktion definiert. Frühes Erkennen und sofortige immunsuppressive Behandlung verbessern die Prognose. Die Nierenbiopsie und die Autoantikörperserologie ermöglichen die genaue diagnostische Zuordnung der RPGN. Es werden ihre Einteilung aufgrund der Immunhistologie, der antineutrophil zytoplasmatische Antikörper (ANCA) und der antiglomerulären BasalmembranAntikörper (anti-GBM) diskutiert. Anhand von Ergebnissen randomisiert kontrollierter Studien werden die Möglichkeiten der differenzierten Behandlung erläutert
\end{abstract}

DOI: https://doi.org/10.1007/s11560-006-0055-x

Posted at the Zurich Open Repository and Archive, University of Zurich ZORA URL: https://doi.org/10.5167/uzh-156233

Journal Article

Published Version

Originally published at:

Pechula Thut, M; Hauser, T (2007). Rasch-progrediente Glomerulonephritis. Der Nephrologe, 2(1):27-32. DOI: https://doi.org/10.1007/s11560-006-0055-x 
Nephrologe 2007 · 2:27-32

DOI 10.1007/s11560-006-0055-x

Online publiziert: 3 . Januar 2007

(c) Springer Medizin Verlag 2007

Redaktion

R.P. Wüthrich, Zürich
M. Pechula Thut ${ }^{1} \cdot$ T. Hauser ${ }^{2}$

${ }^{1}$ Klinik für Nephrologie, Universitätsspital Zürich

${ }^{2}$ Klinik für Immunologie, Universitätsspital Zürich

\section{Rasch-progrediente Glomerulonephritis}

\section{Bewährte und neuere Therapieansätze}

Die rasch progrediente Glomerulonephritis (RPGN) ist eine Nierenerkrankung, die nach frühem Erkennen und schnellem Therapiebeginn oft erfolgreich behandelt werden kann. Die glomerulären Nierenerkrankungen beinhalten ein großes Spektrum an verschiedenen Erkrankungen. Auch wenn die RPGN ein seltenes Syndrom darstellt und mit nur 2-5\% aller $\mathrm{Ne}$ phropathien zu Buche steht, ist es doch sehr wichtig, dieses klinische Syndrom früh zu erkennen. Der Hauptgrund liegt darin, dass bei der RPGN (wie der Name sagt) der Nierenfunktionsverlust rasch über Tage bis Wochen zunimmt. Definitionsgemäß nimmt die glomeruläre Filtrationsrate (GFR) in 3 Monaten um mindestens $\mathbf{5 0 \%}$ ab [15]. Histologisch findet sich eine Glomerulonephritis mit Halbmondbildung (Halbmond-Nephritis) (-Abb. 2) in mehr als $\mathbf{5 0 \%}$ der in der Biopsie vorgefundenen Glomerula. Der Schweregrad der Erkrankung korreliert gut mit der Anzahl der befallenen Glomerula und mit dem Befallsmuster. Sind mehr als $\mathbf{8 0} \%$ der Glomerula befallen, so ist die Nierenfunktion in der Regel stark eingeschränkt und gleichzeitig das Ansprechen auf die Therapie schlechter [16].

\section{Klassifikation}

Die Einteilung [17] erfolgt am übersichtlichsten anhand der immunhistologischen und serologischen Befunde (• Tab. 1).
Typ I: RPGN durch

Anti-GBM-Antikörper

Bei dieser Erkrankung finden sich im Serum antiglomeruläre Basalmembran-Antikörper (anti-GBM). Diese führen in der Niere zu der Erkrankung im Sinne einer RPGN und in der Lunge allenfalls zu einer Kapillaritis mit Alveolarhämorrhagie. Dieses pulmorenale Syndrom wird auch mit dem Begriff Goodpasture-Syndrom bezeichnet. In der Immunfluoreszenz der Nierenbiopsie finden sich die für dieses Syndrom pathognomonischen Anti-GBM-Ablagerungen (• Abb. 1).

Typ II: RPGN durch Immunkomplexe

Die Immunkomplexnephritis (glomeruläre Immunablagerungen) ist die zweithäufigste der 3 Typen. Sie beinhaltet eine sehr vielfältige Krankheitsgruppe. In dieser Gruppe ist die Halbmondbildung nicht typisch. Wird die Halbmondbildung doch gefunden, spricht sie für eine schwere Verlaufsform bei systemischer, immunkomplexvermittelter Erkrankung. Diese GN findet sich beim systemischen Lupus erythematodes (SLE), bei der Kryoglobulinämie und bei Infektionen. Auch bei primären Glomerulonephritiden wie der IgA-GN, einer membranösen oder einer membranoproliferativen GN können Halbmondbildungen bioptisch nachgewiesen werden. $\mathrm{Zu}$ dieser heterogenen Gruppe gehören auch die im Rahmen von Malignomen, vor allem Lymphomen, auftre- tenden und die durch Medikamente (z. B. Penicillamin) hervorgerufenen RPGN.

\section{Typ III: Pauciimmune RPGN}

Der dritte Typus ist die häufigste Form der RPGN. In über der Hälfte aller Biopsien dieser RPGN liegt die als Halbmondnephritis (CGN, ,crescentic glomerulonephritis“) (• Abb. 2) bezeichnete GN vor. Es gibt dabei keine oder nur sehr wenige Immunablagerungen, weshalb man von einer pauciimmunen GN spricht. Bei mehr als $80 \%$ der Patienten mit pauciimmuner GN finden sich im Serum Antikörper, die an Bestandteile des Zytoplasmas von neutrophilen Granulozyten oder Monozyten binden, die antineutrophilen zytoplasmatischen Antikörper („anti neutrophil cytoplasmic antibodies“, ANCA).

\section{Charakteristiken der ANCA-assoziierten RPGN}

Die ANCA-assoziierte RPGN tritt im Rahmen einer systemischen Kleingefäßvaskulitis auf. Gemäß den Definitionen der Chapel Hill Consensus Conference [14] sind mit kleinen Gefäßen Kapillaren, Venulen und Arteriolen gemeint. Kleingefäßvaskulitiden können auch mittlere Gefäße befallen, Mittel- und Großgefäßvaskulitiden betreffen hingegen definitionsgemäß nur größere Gefäße wie Arterien. Der Begriff ANCA-assoziierte systemische Vaskulitis (AASV) umfasst die Wegener'sche-Granulomatose (WG), das Churg-Strauss Syndrom (CSS) sowie die 


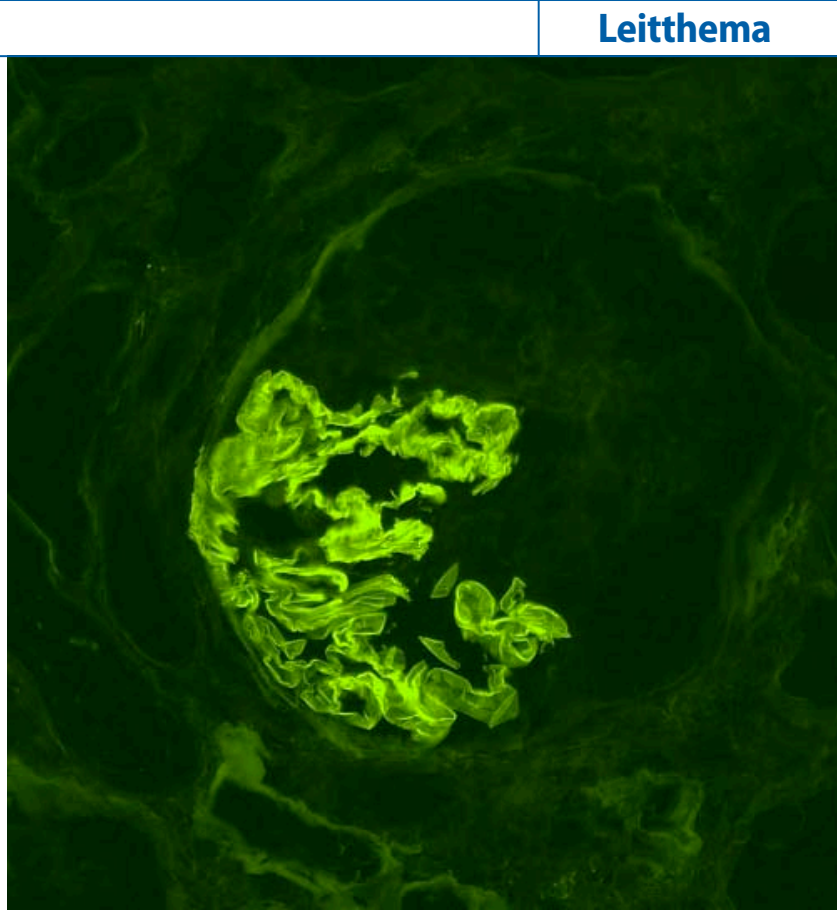

Abb. $1<$ Immunfluoreszenzbild mit linearem Bindungsmuster von IgG an die Basalmembran der glomerulären Kapillarschlingen. (Zur Verfügung gestellt von A. Gaspert, Pathologie, Universitätsspital Zürich)

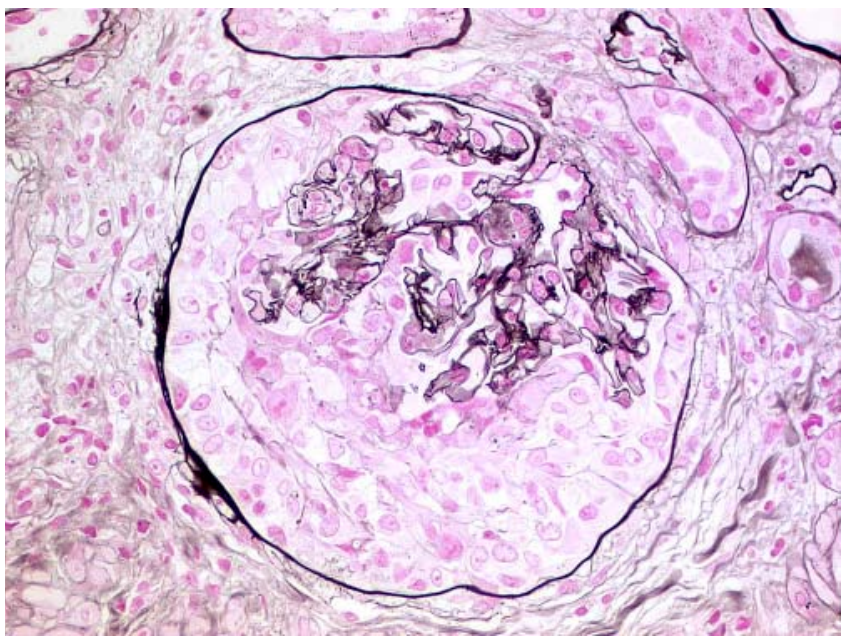

Abb. $2<$ Glomerulus mit globalem zellulären Halbmond und kollabierten Kapillarschlingen in Silbermethamin-Färbung. (Zur Verfügung gestellt von A. Gaspert, Pathologie, Universitätsspital Zürich)

mikroskopische Polyangiitis (MPA) und deren auf die Nieren begrenzte Form. Für epidemiologische Studien steht ein kürzlich erprobter Klassifikationsalgorithmus zur Verfügung, der allerdings wie alle Klassifikationen nicht für die Diagnose beim einzelnen Patienten geschaffen oder validiert wurde [30].

\section{Patienten mit Verdacht auf ANCA-assoziierte GN sind Notfälle}

Die Beschwerden und Befunde von ANCA-assoziierten systemischen Vaskulitiden sind nicht spezifisch, sie überlappen sich mit jenen von Infektionen, Kollagenosen und malignen Tumoren. In vielen Fällen berichten die Patienten von über
Monate anhaltenden Allgemeinsymptomen wie Krankheitsgefühl, Gewichtverlust, Fieber sowie Arthralgien und Myalgien. Die Manifestationen der AASV sind sehr vielfältig, sodass die Syndrome (WG, MPA, CSS) nur selten mit allen ihren klassischen Befunden beobachtet werden können. Dies birgt die Gefahr einer verzögerten Diagnose. Da bei den AASV beinahe alle Organe befallen sein können, ist das Beschwerdebild komplex. Häufig sind Gelenksbeschwerden, Hautveränderungen, Augenentzündungen, Atemwegssymptome sowie Ausfälle der peripheren Nerven. Vorkommen können auch kardiale, gastrointestinale oder zentralnervöse Beteiligungen.

Bei der nur auf die Niere beschränkten AASV kann die Vaskulitis lange unbe- merkt bleiben. In diesem Fall kommt der Kranke häufig erst im Spätstadium zum Arzt. Es können bereits Müdigkeit, Ödeme oder gar ein vollständiges urämisches $\mathrm{Be}$ schwerdebild vorliegen. Hämatologisch bestehen häufig eine Anämie und eine Leukozytose. An pathologischen Laborergebnissen bestehen zudem erhöhte Entzündungsparameter (BSG, CRP), erhöhte Retentionsparameter (Serumkreatinin) und je nach Schweregrad der Niereninsuffizienz Elektrolytstörungen.

Möglichst schnell müssen eine Urinanalyse (Protein, Sediment), serologische Tests (ANCA, Anti-GBM, ANA) sowie eine Nierenbiopsie erfolgen. Patienten mit Verdacht auf ANCA-assoziierte GN sind Notfälle und benötigen in der Regel eine Hospitalisierung mit interdisziplinärer Betreuung.

\section{Labordiagnostik der ANCA}

Die Bestimmung der ANCA beinhaltet zwei Verfahren: die indirekte Immunfluoreszenz (IIF) unter dem Mikroskop und den Enzymimmunoassay (EIA, ELISA). In der indirekten Immunfluoreszenzfärbung werden 3 Bindungsmuster auf fixierten neutrophilen Granulozyten beschrieben: zytoplasmatische (c-ANCA), perinukleäre (p-ANCA) (• Abb. 3) und atypische ANCA. Das c-ANCA-Muster zeigt sich vor allem bei Antikörpern, die Proteinase $3\left(\mathrm{PR}_{3}\right)$ binden. Für Antikörper mit p-ANCA Muster hingegen ist eine Vielfalt intrazellulärer Antigene bekannt; das häufigste und einzige diagnostisch relevante ist die Myeloperoxidase (MPO). In vivo befinden sich sowohl MPO wie auch $\mathrm{PR}_{3}$ in den azurophilen Granula der neutrophilen Granulozyten. Die unterschiedliche intrazelluläre Verteilung der beiden Proteine in der IIF entsteht erst durch Fixierung der Zellen mit Ethanol. Im Enzymimmunoassays werden die Antikörper (PR3-ANCA, MPO-ANCA) durch Bindung an die aufgereinigten Proteine $\mathrm{PR}_{3}$ und MPO bestimmt, die entweder auf eine Mikrotiterplatte oder auf einem Teststreifen aufgetragen sind.

Sensitivität, Spezifität und positive prädiktive Wertigkeit von ANCA sind hoch für die systemische WG, jedoch deutlich geringer für die MPA oder das CSS. Werden c-/PR3- und p-/MPO-ANCA bei Pa- 
tienten mit einer hohen klinischen Vortestwahrscheinlichkeit einer AASV bestimmt, steigt ihre positiv voraussagende Wertigkeit („positive predictive value“), ohne dass sie an Sensitivität verlieren [23]. Diese Marker sollten also nur bei hohem klinischem Verdacht auf eine AASV zur Anwendung kommen. Das kombinierte Ergebnis der IIF und des Enzymimmunoassays ergibt eine höhere Sensitivität des ANCA-Tests [5, 27]. Unter der Voraussetzung einer hohen Vortestwahrscheinlichkeit ist c-ANCA sehr sensitiv für aktive, systemische WG (90-95\%) mit einer Spezifität von 90\% [27]. Hingegen ist p-ANCA weniger sensitiv und kann nur als beschränkter Hinweis auf eine mögliche MPA, renal begrenzte Vaskulitis oder ein CSS dienen; p-ANCA werden nebenbefundlich bei vielen anderen Krankheiten, insbesondere auch bei Infektionen, gefunden [10].

\section{Ätiologie und Pathogenese}

Obwohl die Halbmondbildung allen 3 Typen der RPGN eigen ist, sind weder Ätiologie noch Pathogenese gleich. Die genauen molekularen und zellulären Mechanismen, die zur Halbmondbildung führen, sind noch nicht exakt geklärt. Dennoch kann postuliert werde, dass verschiedene Ursachen in eine gemeinsame pathogenetische Kaskade münden. Dabei führen die ANCA- und Anti-GBM-assoziierten GN zu einer stärkeren Halbmondbildung als die Immunkomplex-GN [16].

Am Anfang der Halbmondbildung steht die Bildung einer Läsion in der glomerulären Kapillarwand und der GBM [3]. Wie mehrere Tiermodelle zeigen konnten, können sowohl die Anti-GBMAntikörper als auch die ANCA diesen Prozess auslösen. Läsionen in der Bowman-Kapsel sind ebenfalls mit der Bildung von Halbmonden vergesellschaftet [19]. Durch die entstandenen Lecks können verschiedene Zellen, Entzündungsmediatoren und Plasmaproteine in den Bowman-Raum eindringen. Dabei sind Fibrin, Makrophagen, glomeruläre Epithelzellen und die interstitiellen Fibroblasten die wichtigsten Auslöser einer Halbmondbildung [29]. Sind aktivierte Makrophagen in den Bowman-Raum gelangt, kommt es zur Zellproliferation. Interleu- kin-1 (IL-1) und Tumornekrosefaktor $\alpha$ (TNFa) spielen eine wichtige Rolle bei der Pathogenese [19]: Sie führen zur Expression von Adhäsionsmolekülen und stimulieren die Zellproliferation. An der darauf folgenden Bildung von fibrozellulären Halbmonden sind Fibroblasten [19] und die parietalen Epithelzellen [25] beteiligt. Neuere Erkenntnisse haben ergeben, dass auch Podozyten an der Halbmondbildung beteiligt sein können [1].

\section{Prognose}

Eine unbehandelte RPGN führt in der Regel innerhalb von Wochen bis Monaten $\mathrm{zu}$ einer terminalen Niereninsuffizienz. Generell gilt: Je mehr Halbmonde in der Nierenbiopsie vorgefunden werden, desto schlechter ist die Prognose. Finden sich bei AASV mehr fibrosierte als zelluläre Halbmonde, ist die Prognose ebenfalls schlechter [8]. Jennette [16] dokumentierte, dass bei $85 \%$ aller Anti-GBM-Erkrankungen das Serumkreatinin $>857 \mu \mathrm{mol} / \mathrm{l}$ und bei 50\% der ANCA-assoziierten RPGN $>575 \mu \mathrm{mol} / \mathrm{l}$ liegt, bei der ImmunkomplexRPGN hingegen bei nur 13\% der Patienten $>433 \mu \mathrm{mol} / \mathrm{l}$. Das Einjahresüberleben der Patienten bzw. der Nieren liegt bei der Anti-GBM-GN mit entsprechender Therapie (Immunsuppression und Plasmapherese) bei einem Kreatinin $<500 \mu \mathrm{mol} /$ 1 bei 100\% bzw. 95\%. Liegt das Serumkreatinin $>500 \mu \mathrm{mol} / \mathrm{l}$, fallen diese Werte auf $83 \%$ bzw. $82 \%$ ab. Ist hingegen bereits eine Nierenersatztherapie bei Diagnosestellung notwendig, fallen das Einjahresüberleben des Patienten und der Niere auf 65\% und $8 \%$ ab [20]. Bei $5 \%$ aller ANCA-positiven GN treten gleichzeitig Anti-GBMAntikörper im Serum auf. Dagegen sind $32 \%$ aller Anti-GBM-AK-GN auch positiv für ANCA. Bei einer Erkrankung mit Anti-GBM-AK und ANCA ist die Prognose entsprechend dem Verlauf einer Anti-GBM-GN, also schlechter als bei einer ANCA-assoziierten Erkrankung [21]. Betrachtet man die AASV über mehr als 8 Jahre, so haben die PR3-ANCA-Erkrankung bzw. die WG betreffend Nieren- und Patientenüberleben die schlechteste Prognose im Vergleich zu ANCA-negativen oder MPO-ANCA-positiven Erkrankungen. Gleichzeitig sind eine terminale Niereninsuffizienz, ein Alter $>68$ Jahren,
Nephrologe 2007 · 2:27-32

DOI 10.1007/s11560-006-0055-x

○) Springer Medizin Verlag 2007

\section{Pechula Thut $\cdot$ T. Hauser Rasch-progrediente Glomerulonephritis. Bewährte und neuere Therapieansätze}

\section{Zusammenfassung}

Die rapid progrediente Glomerulonephritis (RPGN) ist als klinisches Syndrom durch die zeitliche Veränderung der Nierenfunktion definiert. Frühes Erkennen und sofortige immunsuppressive Behandlung verbessern die Prognose. Die Nierenbiopsie und die Autoantikörperserologie ermöglichen die genaue diagnostische Zuordnung der RPGN. Es werden ihre Einteilung aufgrund der Immunhistologie, der antineutrophil zytoplasmatische Antikörper (ANCA) und der antiglomerulären Basalmembran-Antikörper (anti-GBM) diskutiert. Anhand von Ergebnissen randomisiert kontrollierter Studien werden die Möglichkeiten der differenzierten Behandlung erläutert.

\section{Schlüsselwörter}

RPGN · ANCA-assoziierte Glomerulonephritis - Vaskulitis - Immunsuppressiva - Plasmapherese

\section{Rapid progressive glomerulonephritis. Classical and new therapy options}

\section{Abstract \\ Rapid progressive glomerulonephritis (RPGN) is defined as a clinical syndrome based on the change of renal function over time. Early re- cognition and immediate immunosuppres- sive intervention improve renal and general outcomes. Kidney biopsy and autoantibody serology are necessary to determine the pre- cise diagnosis of the disease leading to RPGN. The classification of RPGN is based on immu- nohistology, anti-neutrophil cytoplasmic an- tibodies (ANCA) and anti-glomerular base- ment membrane antibodies (anti-GBM). Re- sults of randomised controlled trials are put into the perspective of adapting therapy to clinical findings.}

\section{Keywords}

RPGN · ANCA-associated glomerulonephritis - Vasculitis · Immunosuppression · Plasma exchange 
perinukleäre

(p)-ANCA

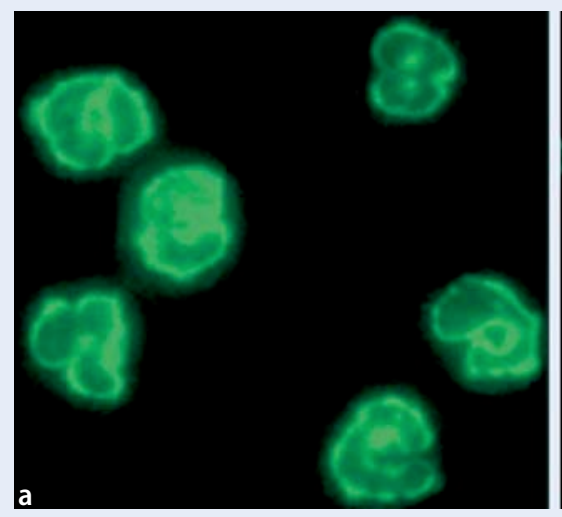

zytoplasmatische

(c)-ANCA

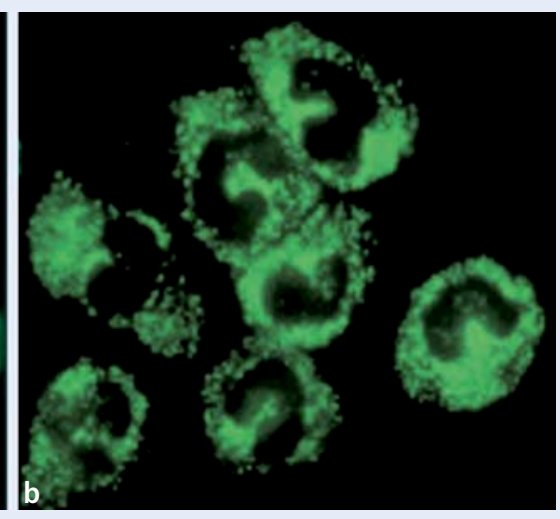

Abb. $3 \Delta$ Immunfluoreszenzbild von ANCA auf neutrophilen Granulozyten. a perinukleäre (p-ANCA), b zytoplasmatische (c-ANCA)

ein Serumkreatinin $>580 \mu \mathrm{mol} / \mathrm{l}$ und eine GFR $<11 \mathrm{ml} / \mathrm{min}$ bei Diagnosestellung mit der schlechtesten Prognose nach 96 Monaten vergesellschaftet [31].

\section{Behandlung}

Das schnelle Erkennen des Krankheitsbildes und die rasche Kontrolle der Krankheitsaktivität mittels hochdosierter Immunsuppressiva sind die wichtigsten Grundsätze der Behandlung der RPGN, um einer bleibenden Schädigung der Niere vorzubeugen. Als empirische Therapie der RPGN hat sich die sofortige i.v. Gabe von Methylprednisolon etabliert. Auch bei einer erst kurze Zeit später entnommenen Nierenbiopsie wird der histologische Befund durch das Kortikosteroid nicht dahingehend verändert, dass die Diagnose verfälscht würde. Bei gleichzeitigem Auftreten einer Alveolarblutung, d. h. dem pulmorenalen Syndrom, ist die Plasmapherese indiziert.

\section{Goodpasture-Syndrom}

Bei der Anti-GBM-Antiköper Erkrankung (Goodpasture-Syndrom) gilt als Therapie der Wahl der Einsatz der Plasmapherese in Kombination mit Kortikosteroid und Cyclophosphamid. Dabei werden durch die Plasmapherese die Anti-GBM-Antikörper und vermutlich weitere Entzündungsmediatoren wie etwa Komplementproteine entfernt. Diese gut etablierte Therapie wurde in nichtkontrollierten Studien untersucht. In nur einer randomi- siert kontrollierten Studie mit lediglich 17 Patienten war die Plasmapherese der alleinigen Gabe von Immunsuppressiva überlegen [17]. Es zeigte sich auch, dass ein geringerer histologischer Befall und ein Kreatininwert von $<265 \mu \mathrm{mol} / \mathrm{l}$ prognostisch pherese wird in der Regel über 2-3 Wochen täglich oder alle 2 Tage durchgeführt und wird solange fortgesetzt, bis die Serumkonzentration der Anti-GBM-AK stabil niedrig bleibt.

Die immunsuppressive Therapie wird meistens mit Kortikosteroidstößen begonnen. Nach dreimaliger Gabe von Methylprednisolon zu $1 \mathrm{~g} / \mathrm{Tag}$ wird auf hochdosiertes perorales Prednison $(1 \mathrm{mg} / \mathrm{kg}$ Körpergewicht) gewechselt. Eine langsame Reduktion erfolgt nach gutem Ansprechen. Das Cyclophosphamid wird in der Regel peroral ( $2 \mathrm{mg} / \mathrm{kg} \mathrm{KG}$ ) appliziert. Nach 2-3 Monaten kann empirisch auf weniger toxische Substanzen wie Azathioprin gewechselt werden. Insgesamt dauert die Therapie in der Regel 6-9, maximal 12 Monate [20]. Verlaufskontrollen der Anti-GBM-AK im Serum sind initial vor jeder Plasmapherese und später alle 24 Wochen nötig.

\section{ANCA-assoziierte systemische Vaskulitiden}

Die Therapie AASV wird in zwei Phasen unterteilt. Während einer anfänglichen Phase der Remissionsinduktion mit hochdosierter, kombinierter Immunsuppression wird der aktive Krankheitsprozess begünstige Indikatoren sind. Die Plasma- herrscht. Darauf folgt eine Phase der Remissionserhaltung, in der eine weniger intensive Therapie eingesetzt wird, um Rückfälle zu verhindern und das Risiko von unerwünschten Arzneimittelnebenwirkungen zu verringern.

Die Induktionstherapie richtet sich nach dem Schweregrad der Erkrankung. Die Europäische Vaskulitis Studiengruppe (European Vasculitis Study Group, EUVAS) [33] schlägt pragmatisch 5 Kategorien nach Schweregrad vor (• Tab. 2). Dabei wird das Vorhandensein von Allgemeinsymptomen, drohendes Versagen von vitalen Organen und das Ausmaß der Niereninsuffizienz herangezogen [11].

Bei der begrenzten und frühen, generalisierten Erkrankung liegt das Serumkreatinin $<120 \mu \mathrm{mol} / \mathrm{l}$, es sind keine anderen vitalen Organe wie die Lunge gefährdet. Die randomisiert kontrollierte Studie NORAM konnte frühere Berichte bestätigen, indem sie zeigte, dass Methotrexat und Cyclophosphamid jeweils kombiniert mit oralem Kortikosteroid gleichwertig sind, um die Remission von früher, generalisierter AASV zu erreichen [7]. Somit kann Methotrexat als wirksame und weniger toxische Alternative verwendet werden, vorausgesetzt es wird unter Berücksichtigung der GFR genügend hoch dosiert. Zur verbesserten Bioverfügbarkeit empfiehlt sich die subkutane Administration einmal pro Woche.

Bei aktiver, generalisierter Erkrankung wird seit der wegweisenden Studie von Fauci et al. [9] orales Cyclophosphamid zusammen mit oralem Kortikosteroid eingesetzt. Neuere Berichte insbesondere über die vorläufigen Resultate der randomisiert kontrollierten Studie CYCLOPS deuten aber darauf hin, dass die i.v. Verabreichung von Cyclophosphamid ( $15 \mathrm{mg} / \mathrm{kg} \mathrm{KG)}$ in erst 2-, dann 3-wöchigen Abständen während 3-6 Monaten ebenso wirksam ist wie die tägliche orale Gabe, allerdings mit dem Vorteil geringerer unerwünschter Arzneimittelwirkungen [6]. Die i.v. Verabreichungsweise hat sich in den laufenden Studien der EUVAS als Standardtherapie der Kontrollgruppen durchgesetzt [33]. Mycophenolatmofetil wird zur Zeit im Vergleich zu Cyclophosphamid in der großen randomisiert kontrollierten Studie MYCYC untersucht [33]. Die gegen TNFa gerichtete Therapie 
mit Etanercept war in der einzigen bisherigen randomisierten, placebokontrollierten Studie bei WG unwirksam [31].

Die schwere Erkrankung bei AASV mit einem Serumkreatinin von $>500 \mu \mathrm{mol} / \mathrm{l}$ bedarf einer intensiveren Behandlung. Die randomisiert kontrollierte Studie MEPEX verglich i.v. Methylprednisolon $3 \times 1$ g/Tag mit Plasmapherese $7 \times 4$ l über 3 Wochen als anfängliche Zusatztherapie zu Cyclophosphamid und oralem Kortikosteroid bei 137 Patienten. Das Überleben sowohl der Patienten als auch der Nieren war nach $3 \mathrm{Mo}-$ naten wie auch einem Jahr deutlich besser in der Gruppe mit Plasmapherese [13].

\section{Zur Therapie von refraktärer Erkrankung bei progredientem Verlauf wiesen Infliximab als auch ein Anti-Thymozyten-Globulin eine hohe Ansprechrate auf}

Zur Therapie von refraktärer Erkrankung bei progredientem Verlauf unter der beschriebenen Therapie gibt es zurzeit nur entweder nichtkontrollierte Studien oder solche mit kleinen Patientenzahlen. Allerdings wiesen sowohl der Anti-TNFa-Antikörper Infliximab [4], als auch ein Anti-Thymozyten-Globulin eine hohe Ansprechrate auf [28]. Vielversprechend sind die Resultate einer kleinen Serie von AASV, bei welcher die Behandlung mit dem Anti-CD2o-Antikörper Rituximab zusammen mit Kortikosteroid bei allen elf Patienten zur Remission führte [18]. Zurzeit untersuchen zwei randomisierte, kontrollierte Studien verschiedene Behandlungsschemata mit Rituximab. Die eine Studie untersucht Rituximab zusammen mit Kortikosteroid im Vergleich zur Standardtherapie aus Kortikosteroid, Cyclophosphamid und Azathioprin. Die andere, RITUXVAS, prüft die Dreierkombination Rituximab, Kortikosteroid und nur 2 i.v. Dosen Cyclophosphamid im Vergleich zum Standard; damit soll der vermutlich verzögerten Wirkung von Rituximab auf die RPGN Rechnung getragen werden [33].

\section{- Das Rezidivrisiko der AASV ist hoch}

Nach Erreichen der Remission innerhalb meistens 3-6 Monaten soll auf eine Er-

\begin{tabular}{|clll|}
\hline Tab. 1 & Einteilung der RPGN & Serologie & Grunderkrankung \\
\hline Typ I & $\begin{array}{l}\text { Immunhistologie } \\
\text { Ig-Ablagerungen entlang der } \\
\text { glomerulären Basalmembran }\end{array}$ & Anti-GBM-Antikörper & $\begin{array}{l}\text { Anti-GBM-Nephritis, } \\
\text { Goodpasture-Syndrom (mit } \\
\text { Lungenbefall) }\end{array}$ \\
\hline Typ II & $\begin{array}{l}\text { Ig-Ablagerungen mesangial } \\
\text { und/oder kapillär }\end{array}$ & $\begin{array}{l}\text { C3, C4, ANA, Anti-DNS-AK, } \\
\text { Anti-Streptokokken-AK, } \\
\text { Kryoglobuline }\end{array}$ & $\begin{array}{l}\text { Systemischer Lupus erythe- } \\
\text { matodes, postinfektiöse GN } \\
\text { andere GN (IgA-GN, memb- } \\
\text { ranöse GN, membranopro- } \\
\text { liferative) }\end{array}$ \\
Typ III & $\begin{array}{l}\text { Pauciimmun: keine oder nur } \\
\text { wenige Ablagerungen }\end{array}$ & $\begin{array}{l}\text { c-/PR3-ANCA } \\
\text { p-/MPO-ANCA }\end{array}$ & $\begin{array}{l}\text { ANCA-assoziierte syste- } \\
\text { mische Vaskulitiden (AASV) }\end{array}$ \\
\hline Ig Immunglobulin;C Komplementprotein; AK Antikörper. & \\
\hline
\end{tabular}

Tab. 2 Etablierte Therapien der AASV abgestimmt auf den Schweregrad. (Mod. nach

[11])

\begin{tabular}{|lllll}
\hline & $\begin{array}{l}\text { Serumkreatinin } \\
(\mu \mathrm{mol} / \mathrm{l})\end{array}$ & $\begin{array}{l}\text { Vitalorgane be- } \\
\text { droht }\end{array}$ & $\begin{array}{l}\text { Remissions- } \\
\text { induktion }\end{array}$ & $\begin{array}{l}\text { Remissions- } \\
\text { erhaltung }\end{array}$ \\
\hline $\begin{array}{l}\text { Begrenzt } \\
\text { Früh generalisiert }\end{array}$ & $>120$ & Nein & $\begin{array}{l}\text { KS und/oder MTX } \\
\text { KS }+ \text { MTX oder KS } \\
+ \text { CYC }\end{array}$ & $\begin{array}{l}\text { KS + AZA oder KS } \\
+ \text { MTX }\end{array}$ \\
$\begin{array}{l}\text { Aktiv generali- } \\
\text { siert }\end{array}$ & $<500$ & Ja & KS + CYC & KS + AZA \\
\hline $\begin{array}{l}\text { Schwer } \\
\text { Refraktär }\end{array}$ & $>500$ & Ja & KS + CYC+PP & KS + AZA \\
\hline Nicht relevant & Ja & Nicht definiert & Nicht definiert
\end{tabular}

KS Kortikosteroide; MTX Methotrexat; CYC intravenöses Cyclophosphamid; AZA Azathioprin; PP Plasmapherese.

haltungstherapie umgestellt werden, die weniger immunsuppressiv ist und weniger Nebenwirkungen mit sich bringt. Die große randomisiert kontrollierte Studie CYCAZEREM [12] zeigte den Nutzen von Azathioprin zusätzlich zu niedrigdosiertem Kortikosteroid in der Erhaltungsphase, nachdem die Remission mit Cyclophosphamid erreicht worden war. Methotrexat könnte gemäß einer nichtkontrollierten Studie in der Erhaltungstherapie ebenso wirksam sein [22]. Sowohl Mycophenolatmofetil [26] als auch Leflunomid [24] wurden als Erhaltungstherapeutika in kleinen nichtkontrollierten Studien eingesetzt und werden in laufenden randomisiert kontrollierten Studien geprüft.

Falls kein Rezidiv auftritt, wird die Behandlung mit Azathioprin im Allgemeinen bis mindestens 18-24 Monate nach Remission weitergeführt. Die Ergebnisse zweier laufender prospektiver Langzeitstudien, REMAIN und IMPROVE, werden genauere Anhaltspunkte zur notwendigen Dauer der Erhaltungstherapie geben [33].

\section{Opportunistische Infekte}

Opportunistische Infekte sind für einen großen Teil der Morbidität und Mortalität unter immunsuppressiver Therapie verantwortlich. Zur Vorbeugung der Pneumocystis-jiroveci-Pneumonie (früher: P. carinii) ist Cotrimoxazol oder bei Sulfonamid-Unverträglichkeit eine alternative Medikation indiziert, solange Cyclophosphamid und hochdosiertes Kortikosteroid verabreicht werden. Gelegentlich wird eine Prophylaxe gegen oropharyngeale Candidose durchgeführt.

Die langfristige Anwendung von Kortikosteroiden erfordert eine adäquate Osteoporoseprophylaxe.

\section{Fazit für die Praxis}

Die Definition der RPGN anhand von einfachen Nierenfunktionsparametern in Serum und Urin soll ihr frühes Erkennen durch den Grundversorger gewährleisten. Die rasche Zuweisung zur interdisziplinären, fachärztlichen Betreuung ermöglicht die genaue Diagnose und eine angepasste Behandlung. Eine sofortige immunsuppressive Therapie ist notwen- 
dig, um die ansonsten schlechte Prognose zu verbessern. ANCA und Anti-GBMAK können eine Diagnose der zugrundeliegenden Krankheiten unterstützen, die letztlich aber durch die Nierenbiopsie bestätigt werden muss.

Unter Berücksichtigung der besten aktuell zur Verfügung stehenden Evidenz können für die Behandlung der RPGN Kortikosteroide, Cyclophosphamid, Azathioprin, Methotrexat und die Plasmapherese eingesetzt werden. Sie müssen auf die zugrundeliegende Erkrankung, deren Schweregrad und die Therapiephase abgestimmt werden. Laufende randomisiert kontrollierte Studien werden die Grundlage für den Einsatz neuerer Immunsuppressiva liefern.

\section{Korrespondierender Autor Dr. T. Hauser}

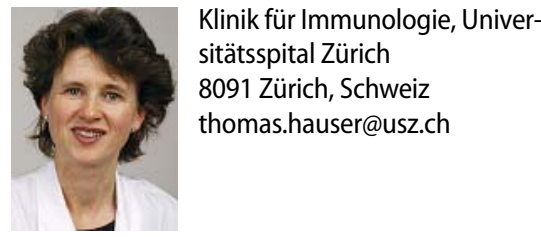

Interessenkonflikt. Es besteht kein Interessenkonflikt. Der korrespondierende Autor versichert, dass keine Verbindung mit einer Firma, deren Produkt in dem Artikel genannt ist, oder einer Firma, die ein Konkurrenzprodukt vertreibt, bestehen. Die Präsentation des Themas ist unabhängig und die Darstellung der Inhalte produktneutral.

\section{Literatur}

1. Atkins RC, Nikolic-Paterson DJ, Song Q, Lan HY (1996) Modulators of crescentic glomerulonephritis. J Am Soc Nephrol 7: 2271-2278

2. Bariety J, Bruneval P, Meyrier A et al. (2005) Podocyte involvement in human immune crescentic glomerulonephritis. Kidney Int 68: 1109-1119

3. Bonsib S (1985) Glomerular basement membrane discontinuities: Scanning electron microscopic study of acellular glomeruli. Am J Pathol 119: 357360

4. Booth A, Harper L, Hammad T et al. (2004) Prospective study of TNFalpha blockade with infliximab in anti-neutrophil cytoplasmic antibody-associated systemic vasculitis. J Am Soc Nephrol 15: 717-720

5. Csernok E, Holle J, Hellmich B et al. (2004) Evaluation of capture ELISA for detection of antineutrophil cytoplasmic antibodies directed against proteinase 3 in Wegener's granulomatosis: first results from a multicentre study. Rheumatology (Oxford) 43: 174-180

6. De Groot K, Adu D, Savage CO, EUVAS (European vasculitis study group) (2001) The value of pulse cyclophosphamide in ANCA-associated vasculitis: meta-analysis and critical review. Nephrol Dial Transplant 16: 2018-2027
7. De Groot K, Rasmussen N, Bacon PA et al. (2005) Randomized trial of cyclophosphamide versus methotrexate for induction of remission in early systemic antineutrophil cytoplasmic antibody-associated vasculitis. Arthritis Rheum 52: 2461-2469

8. De Lind van Wijngaarden RA, Hauer HA, Wolterbeek $R$ et al. (2006) Clinical and histologic determinants of renal outcome in ANCA-associated vasculitis: A prospective analysis of 100 patients with severe renal involvement. J Am Soc Nephrol 17: 2264-2274

9. Fauci AS, Haynes BF, Katz P, Wolff SM (1983) Wegener's granulomatosis: prospective clinical and therapeutic experience with 85 patients for 21 years. Ann Intern Med 98: 76-85

10. Hagen EC, Daha MR, Hermans J et al. (1998) Diagnostic value of standardized assays for anti-neutrophil cytoplasmic antibodies in idiopathic systemic vasculitis. EC/BCR Project for ANCA Assay Standardization. Kidney Int 53: 743-753

11. Jayne D (2000) Evidence-based treatment of systemic vasculitis. Rheumatology (Oxford) 39: 585-595

12. Jayne D, Rasmussen N, Andrassy K et al. (2003) A randomized trial of maintenance therapy for vasculitis associated with antineutrophil cytoplasmic autoantibodies. N Engl J Med 349: 36-44

13. Jayne D, Rasmussen N, Andrassy K et al. (2007) A randomized trial of plasma exchange versus methyl prednisolone for severe renal vasculitis. N Engl J Med

14. Jennette JC, Falk RJ, Andrassy K et al. (1994) Nomenclature of systemic vasculitides. Proposal of an international consensus conference. Arthritis Rheum 37: 187-192

15. Jennette JC, Thomas DB (2001) Crescentic glomerulonephritis. Nephrol Dial Transplant 16: 80-82

16. Jennette JC (2003) Rapidliy progressive crescentic glomerulonephritis. Kidney Int 63: 1164-1177

17. Johnson JP, Moore J Jr, Austin HA 3rd, Balow JE (1985) Therapy of anti-glomerular basement membrane antibody disease: analysis of prognostic significance of clinical, pathologic and treatment factors. Medicine (Baltimore) 64: 219227

18. Keogh KA, Ytterberg SR, Fervenza FC et al. (2006) Rituximab for refractory Wegener's granulomatosis: report of a prospective, open-label pilot trial. Am J Respir Crit Care Med 173: 180-187

19. Lan HY, Nikolic-Paterson DJ, Atkins RC (1992) Involvement of activated periglomerular leucocytes in the rupture of Bowmwn's capsule and crescent progression in expeimental glomerulonephritis. Lab Invest 67: 743-759

20. Levy JB, Turner AN, Rees AJ, Pusey CD (2001) Long-term outcome of anti-glomerular basement membrane antibody disease treated with plasma exchange and immune suppression. Ann Intern Med 134: 1033-1042

21. Levy JB, Hammad T, Coulthart A et al. (2004) Clinical features and outcome of patients with both ANCA and anti-GBM antibodies. Kidney Int 66: 1535-1540

22. Langford CA, Talar-Williams C, Barron KS, Sneller MC (2003) Use of a cyclophosphamide-induction methotrexate-maintenance regimen for the treatment of Wegener's granulomatosis: extended follow-up and rate of relapse. Am J Med 114: 463469

23. Mandl LA, Solomon DH, Smith EL et al. (2002) Using antineutrophil cytoplasmic antibody testing to diagnose vasculitis: can test-ordering guidelines improve diagnostic accuracy? Arch Intern Med 162: 1509-1514
24. Metzler C, Fink C, Lamprecht P et al. (2005) Maintenance of remission with leflunomide in Wegener's granulomatosis. Rheumatology (Oxford) 43: 315320

25. Nitta K, Horita S, Honda K et al. (1999) Glomerular expression of cell-cycle-regulatory proteins in human cresentic gloerulonephritis. Virchows Arch 435: 422-427

26. Nowack R, Gobel U, Klooker P et al. (1999) Mycophenolate mofetil for maintenance therapy of Wegener's granulomatosis and microscopic polyangiitis: a pilot study in 11 patients with renal involvement. J Am Soc Nephrol 10: 1965-1971

27. Russell KA, Wiegert E, Schroeder DR et al. (2002) Detection of anti-neutrophil cytoplasmic antibodies under actual clinical testing conditions. Clin Immunol 103: 196-203

28. Schmitt WH, Hagen EC, Neumann I et al. (2004) Treatment of refractory Wegener's granulomatosis with antithymocyte globulin (ATG): an open study in 15 patients. Kidney Int 65: 1440-1448

29. Tipping PG, Holdsworth SR (1986) The participation of macrophages, glomerular procoagulant activity and factor VIII in glomerular fibrin deposition: Studies in anti-glomerular basement membrane antibody induced glomerulonephritis in rabbits. Am J Pathol 124: 10-17

30. Watts R, Lane S, Hanslik T et al. (2006) Development and validation of a consensus methodology for the classification of the ANCA-associated vasculitides and polyarteritis nodosa for epidemiological studies. Ann Rheum Dis

31. Wegener's Granulomatosis Etanercept Trial (WGET) Research Group (2005) Etanercept plus standard therapy for Wegener's granulomatosis. N Engl J Med 352: 351-361

32. Weidner S, Geuss S, Hafezi-Rachti S et al. (2004) ANCA-associated vasculitis with renal involvement: an outcome analysis. Nephrol Dial Transplant 66: 1403-1411

33. European Vasculitis Study Group (2006) http:// www.vasculitis.org 